https://idp.uoc.edu

ARTÍCULO

\title{
El impacto de la tecnología blockchain en la contratación privada: ¿hacia una contratación inteligente?
}

\author{
Rosa Barceló Compte \\ Universidad de Barcelona
}

Fecha de presentación: octubre de 2020

Fecha de aceptación: abril de 2021

Fecha de publicación: octubre de 2021

\section{Resumen}

El presente trabajo analiza, esencialmente, el impacto de la tecnología blockchain en el derecho contractual mediante los Ilamados «contratos inteligentes» y su encaje en el ordenamiento jurídico. Así, se aborda el concepto de smart contracts y se examinan las principales características de la tecnología blockchain y del contrato inteligente, así como los límites sustantivos propios de estos contratos.

En este sentido, se presentan las diferencias con la contratación tradicional y se proponen mecanismos de mejora con relación a los contratos inteligentes. De igual modo, se contempla específicamente la cuestión relativa al consentimiento prestado por las partes en esta forma de contratación y se establece la necesidad de intervención notarial en aras a la protección de dicho consentimiento.

Finalmente, se plantea un modelo de arbitraje inteligente que permita paliar el efecto de automatismo propio de los smart contracts

\section{Palabras clave}

contratos inteligentes, blockchain, arbitraje, derecho contractual, consumidor, consentimiento 


\title{
The impact of blockchain technology in private contracting: towards intelligent contracting?
}

\begin{abstract}
The present study investigates the impact of blockchain technology into contractual law through the so-called smart contracts and its place within the legal system. Thus, the concept of smart contracts is approached and the main characteristics of blockchain technology and smart contracts are examined, as well as the substantive limits of these contracts.

To that end, the differences with traditional contracts are presented and, in relation to smart contracts, upgrades mechanisms are suggested. Likewise, the given consent by the parties within this form of contracting is discussed and the necessary notarial intervention is laid down with the goal of protecting that consent.

Ultimately, a smart arbitration model is proposed in order to relieve the automation effect of smart contracts.
\end{abstract}

\section{Keywords}

intelligent contracts, blockchain, arbitration, contract law, consumer, consent 


\section{Introducción y objetivo del artículo}

El derecho de los contratos ocupa un lugar central en los planes de estudio de las universidades de Derecho y en la práctica del derecho en cualquier ordenamiento jurídico'. Por ello, y citando al profesor Salvador Coderch², «no es extraño que cada cierto tiempo reciba los ataques de quienes preconizan sustituirlo, ya sea por sistemas centralizados y jerárquicos, cardinalmente, de derecho público, ya sea por sistemas descentralizados, autogestionados al margen de toda autoridad central».

Pues bien, los llamados «contratos inteligentes» (smart contracts) pretenden desplazar el ya tradicional derecho contractual. El crecimiento y la consolidación de la tecnología de la cadena de bloques (blockchain) ha refrendado la contratación inteligente como la protagonista jurídica de la revolución digital, junto con la inteligencia artificial. Este trabajo pretende abordar, precisamente, la aplicación de dicha tecnología en el ámbito de la contratación privada, así como poner el acento en la dimensión contractual de los smart contracts y proponer pautas de mejora en el diseño de estos contratos que les permitan superar aquellas barreras que, por sus características de automatismo $^{3}$ e inmutabilidad ${ }^{4}$, pueden lastrar el desarrollo de la digitalización en el ámbito contractual.

\section{La tecnología blockchain: ¿The Trust Machine?}

De forma muy genérica, podemos definir la tecnología blockchain como una base de datos descentralizada que es mantenida por una red distribuida de ordenadores ${ }^{5}$, aunque, como señala MIK, no hay una definición universal' ${ }^{6}$. Las características esenciales de esta tecnología son su certeza, seguridad, inmutabilidad ${ }^{7}$ e irrefutabilidad. Como resultado final, la tecnología blockchain excluye cualquier intermediario. Técnicamente, la cadena de bloques es un registro de transacciones; sin embargo, se utiliza la misma como plataforma que permite, precisamente, dichas transacciones. Es decir, la blockchain, por sí, actúa como registro compartido e identifica, inequívocamente, los datos anotados. Por lo tanto, es un registro permanente de un volumen mayúsculo de información disponible para todos los asociados a la red ${ }^{8}$.

Desde un punto de vista técnico, este sistema basado en el consenso y la confianza se construye desde una red global de ordenadores que gestionan una ingente base de datos 9,10 .

La aplicación más famosa de la cadena de bloques es la moneda digital bitcoin. Sin embargo, el potencial de la

1. Este trabajo se enmarca en el Proyecto PID2019-107195RB-I00 «Cumplimiento de los contratos y realidad digital: la adaptación del derecho contractual para la prevención de conflictos transfronterizos», IPs: Dra. Mariló Gramunt Fombuena y Dra. Gemma Rubio Gimeno; y en las actividades del Grup de Recerca consolidat Dret civil català i dret privat europeu (2017 SGR 997).

2. Salvador Coderch (2020).

3. El automatismo o self-execution significa que el contrato inteligente se ejecuta automáticamente mediante código informático, sin que haya, por tanto, intervención ni control humano. Al respecto, véase Meyer (2020, pág. 17). Por lo tanto, las consecuencias del acuerdo se desencadenarán cuando se cumpla el requisito previo establecido: Górriz López (2017, páq. 190).

4. Las transacciones operadas en la cadena de bloques no pueden ser modificadas $y$, en consecuencia, en principio es imposible cambiar los términos del contrato. Véase Weber (2019).

5. Véase De Filippi y Wright (2018, pág. 13). También Ibáñez Jiménez (2018, págs. 31-32). Y Nakamoto (2008).

6. Así, Mik (2019, pág. 162).

7. Sin embargo, Mik (2019, pág. 172) señala que la inmutabilidad de lo almacenado por la cadena de bloques se asocia, incorrectamente, a su veracidad y autenticidad.

8. Díaz Díaz (2019, pág. 3)

9. Werbach (2016, pág. 497).

10. La descentralización señalada como ventaja plantea, desde el punto de vista del Derecho, una cuestión relativa a la responsabilidad: ¿qué ocurre cuando el sistema falla? ¿Podríamos hablar de una responsabilidad parecida a la de los prestadores de servicios de internet o intermediarios? ¿Recae un deber de diligencia sobre la propia cadena de bloques por la información almacenada? Dicha cuestión escapa al objeto de este trabajo, pero sin duda debe ser tenida en cuenta para investigaciones futuras. Sobre la responsabilidad de los intermediaros en internet, véase Arroyo Amayuelas (2020, págs. 808-837). También, desde un punto de vista de la propiedad intelectual, véase Xalabarder Plantada (2006, págs. 1-15). Podemos afirmar, pues, que en la cadena de bloques no existe un ente central contra quien se pueda ejercer una acción que tenga como objeto la exigencia de dicha responsabilidad por el contenido alojado en la misma; así, por ejemplo, Mik (2019, pág. 165). 
misma ha sido explotado, también, en el contexto de los servicios financieros o en el ámbito de la Administración pública"1. La desvinculación de la tecnología blockchain de la moneda virtual promovió el nacimiento de los contratos inteligentes.

\section{Contratos inteligentes o blockchain-based smart contracts: los iContracts}

En el ámbito del derecho contractual es especialmente interesante por su condición novedosa la idea de «contratación inteligente» desarrollada por Nick Szabo antes del auge de la blockchain y del bitcoin ${ }^{12}$. Este autor señalaba que las relaciones contractuales podían ser insertadas en el seno del hardware y del software ${ }^{13}$. Para Szabo, la máquina expendedora de refrescos dio lugar a la aparición del primer contrato inteligente.

Cabe apuntar aquí que el término smart contract puede llevar al lector a una idea equivocada al integrar en su raíz la palabra «contrato». Así, existirá contrato si se cumplen los requisitos exigidos legalmente para su validez (art. 1261 CCE: consentimiento, objeto y causa); por lo tanto, de entrada, el contrato inteligente puede ser ajeno a la esfera jurídica. Al respecto, distinguiremos entre contratos inteligentes jurídicamente irrelevantes y contratos inteligentes jurídicamente relevantes ${ }^{14}$. Si observamos la definición propuesta por Legerén-Molina relativa a los contratos que se ejecutan en la cadena de bloques, el término smart contract, desde la perspectiva técnica, se relaciona con la secuencia de código informático que recoge las condiciones establecidas por las partes y las consecuencias que se desencadenan cuando se cumplen aquellas condiciones. Por lo tanto, las características principales de los contratos inteligentes son, por una parte, el código informático, y, por otra, su carácter de autoejecución (self-execution) o ejecución automática15.

En el presente artículo nos centraremos en los contratos basados en la cadena de bloques, los blockchain-based smart contracts, a los que también llamaremos, indistintamente, iContracts.

La peculiaridad o característica definitiva de estos contratos es que, como hemos dicho, están diseñados sobre la base de un lenguaje informático y se ejecutan sobre una cadena de bloques (la plataforma que más ha desarrollado la contratación inteligente ha sido Ethereum ${ }^{16}$ ).

Los iContracts funcionan como la condición (if-then) de un programa informático. Tienen, por tanto, una lógica booleana: si se cumple la circunstancia (if), entonces se ejecuta la acción (then); de no cumplirse, se ejecuta otra acción, también prevista por el propio contrato (e/se $)^{17}$. Piénsese, por ejemplo, en un contrato de transporte aéreo: si no se realiza el vuelo programado (if), y no se ha informado al pasajero al menos con dos semanas de antelación con respecto a la hora de salida prevista, (then) entonces se compensaría -de forma automática- económicamente al pasajero cuyo vuelo ha sido cancelado. Si se realiza tal y como estaba programado, no habrá compensación (else).

Con relación a la estructura de los smarts contracts, cabe constatar que no constituye una nueva modalidad contractual; es decir, el smart contract es una forma de contrato electrónico (regulado en nuestro ordenamiento por la Ley 34/2002, de 11 de julio, de Servicios de la Sociedad de la Información y de Comercio Electrónico), escrito en lenguaje informático, con una descentralización de los datos y que se ejecuta automáticamente si se cumplen las condiciones preestablecidas en el mismo ${ }^{18}$. Así, es contrato electrónico todo aquel contrato celebrado por vía electrónica, todo contrato en el que la oferta y la aceptación se transmiten

\footnotetext{
11. Vega Maza (2019, págs. 109-126).

12. Szabo (1997).

13. Szabo (1996).

14. Los contratos inteligentes jurídicamente irrelevantes son los smart code contracts, que constituyen únicamente una mera secuencia de código informático. Véase, al respecto, López Rodríguez (2021, pág. 446).

15. Legerén-Molina (2019, pág. 223).

16. Otras plataformas que han desarrollado contratos inteligentes se mencionan en Zheng et al. (2020, págs. 483-484).

17. Legerén-Molina (2019, loc. cit.).

18. Vilalta Nicuesa (2019, pág. 28).
} 
por medio de equipos electrónicos de tratamiento y almacenamiento de datos, conectados a una red de telecomunicaciones (según la definición establecida por el Anexo único de la Ley 34/2002, de 11 de julio, de Servicios de la Sociedad de la Información y de Comercio Electrónico). En definitiva, el contrato inteligente es un contrato electrónico con un mecanismo de ejecución programado con antelación.

Se diría que, en principio, cualquier persona que tenga capacidad para contratar podría celebrar un contrato mediante la tecnología blockchain y, en este sentido, los smart contracts pueden ser contratos en el sentido expresado por los arts. 1254 y sigs. del CCE ${ }^{19}$. El hecho de que las partes recurran a un medio electrónico, como es la tecnología de la cadena de bloques, condicionará, en cualquier caso, el régimen jurídico (ya que resultarán de aplicación determinadas normas por el uso del soporte electrónico).

Los smart contracts ejecutados en blockchain, así como otros a los que haremos referencia, se benefician de las ventajas de dicha tecnología. Requerirán de un tercero: la propia máquina mediante los oráculos (una red de observadores externos que comprueba que los efectos que se producen en la fase de la ejecución del contrato responden o bien a las instrucciones configuradas por las partes en el sistema o a eventos externos cuya existencia quedará registrada), quienes verificarán si se cumplen las condiciones predeterminadas, desencadenando, así, la eficacia contractual20.

\section{Dimensión contractual del iContract: algunos aspectos relevantes}

Constatamos que no existe en España una regulación específica relativa a aquellos contratos que se ejecutan en una cadena de bloques. Por ello, será la normativa general de obligaciones y contratos la que resultará de aplicación, así como también la específica de la contratación electrónica y la relativa a los servicios de la sociedad de la información ${ }^{21}$.

Además, debemos poner especial atención cuando una de las partes sea un consumidor, ya que entonces entrarán en juego la Ley de Condiciones Generales de la Contratación (Ley 7/1998, de 13 de abril, en adelante LCGC) y el Real Decreto Legislativo 1/2007, de 16 de noviembre, por el que se aprueba el Texto Refundido de la Ley General para la Defensa de los Consumidores y Usuarios y otras leyes complementarias.

A continuación, nos detendremos brevemente en diversas cuestiones que afectan a la formación del contrato inteligente.

\subsection{El consentimiento contractual en los iContracts}

En primer lugar, en la contratación inteligente, el «contrato» es encauzado mediante el código informático. Los iContracts están redactados mediante un lenguaje informático (también Ilamado «formal») que condiciona el desarrollo de los mismos ${ }^{22}$. La cuestión que procede es la siguiente: ¿podemos cuestionar la validez del consentimiento contractual de un iContract formalizado exclusivamente en código informático en la cadena de bloques si no todas las partes intervinientes son expertas en lenguaje de programación?

Por ello, en muchas ocasiones, una de las partes será ajena a la creación del contrato y, en consecuencia, no podrá verificar si el código refleja verazmente lo acordado entre las partes ${ }^{23}$. Necesariamente, la validez de

\footnotetext{
19. El contrato inteligente se verá limitado por el entorno en el que el mismo se celebra y se ejecuta (véase infra §3.2).

20. Legerén-Molina (2018, págs. 196-197).

21. Con relación a la naturaleza contractual del smart contract, véase Argelich Comelles (2020, pág. 12).

22. La distinción entre lenguaje formal y natural la encontramos en Allen (2018, págs. 322-323). Señala dicho autor que la semántica entre ambos lenguajes es muy diferente y que el contenido semántico del lenguaje natural es difícil de formalizar y de ser transformado en código. El lenguaje informático condicionará, pues, la gestación y el desarrollo del contrato y no todos los negocios jurídicos podrán plasmarse como un contrato inteligente. Así lo expresa, también, Idelberger (2018, pág. 216). En un mismo sentido, Górriz López (2017, pág. 189): «no todos los negocios jurídicos podrán configurarse como contratos inteligentes, pues no todos ellos pueden plasmarse en una secuencia de instrucciones [...] ejecutada por un ordenador».

23. Mik (2017, pág. 285)
} 
un contrato legal inteligente exigirá la concurrencia de un doble consentimiento simultáneo: el requerido jurídicamente en el que las partes consienten en obligarse, y el consentimiento preconstituido sobre la consumación automática ${ }^{24}$.

Ciertamente, podemos afirmar que muchos de los smart contracts se originarán a partir de un documento escrito en lenguaje natural que deberá ser traducido a código (lenguaje formal). En un iContract, como en cualquier contrato para que sea válido, hay que cerciorarse de que el consentimiento prestado por las partes es un consentimiento consciente y exacto y, por ello, hay que garantizar la comprensión del código porque la transcripción de este al lenguaje natural y viceversa no será siempre una tarea sencilla ${ }^{25}$. En este sentido, habrá que considerar la incidencia del error como vicio del consentimiento (ex art. 1265 CCE) cuando se produzca una apreciación equivocada de algunos aspectos del contrato, permitiendo a la parte pedir su anulación ${ }^{26}$. Asimismo, cabría plantear si el consentimiento no reside tanto en la comprensión del lenguaje como en la aceptación de la ejecución automática de las prestaciones. El consentimiento de los contratantes hace referencia al acuerdo de voluntades, y ya el art. 1254 CCE señala que «el contrato existe desde que una o varias personas consienten en obligarse, respecto de otra u otras, a dar alguna cosa o a prestar algún servicio». La cuestión residirá, entonces, en determinar si un consentimiento consciente, libre y exacto sobre la ejecución automática es un consentimiento válido de acuerdo con el art. 1261 CCE.

Consideramos que, con el fin de potenciar la seguridad jurídica y la eficiencia de la contratación, un régimen jurídico especial en materia de ejecución de contratos inteligentes debe promover medios de comprobación efectiva de que el consentimiento prestado sea válido y consciente, con conocimiento de causa ${ }^{27}$, en la medida en que el usuario conozca las consecuencias de contratar mediante una red descentralizada ${ }^{28}$ y en la medida en que dicho consentimiento se preste, también, sobre el lenguaje informático.

El interés creciente que surge del potencial que puede llegar a tener la cadena de bloques ha llevado a un sector profano a la comunidad científico-jurídica a afirmar que dicha tecnología suplirá las funciones notariales ${ }^{29}$. El error cometido por entornos no jurídicos identifica la tecnología con la que se presta el servicio con el prestador del servicio en sí: como veremos, la blockchain no realiza un control de legalidad sobre lo almacenado ${ }^{30}$; es solo el medio que facilita la contratación ${ }^{31}$.

En nuestro entorno podemos afirmar que la función notarial se erige como guardián del consentimiento efectivamente prestado por las partes y, con relación a ello, se construye este deber del fedatario público de velar por la protección de la parte más débil del negocio que el mismo autoriza. Como hemos reiterado, el consentimiento que prestan las partes asume la automatización del cumplimiento y ejecución contractual. Nótese que, a este respecto, el notario dará fe del hecho de que los contratantes hayan prestado un consentimiento libre y consciente; además, según ha señalado la doctrina, el notario corrige una particular situación de asimetría contractual ${ }^{32}$.

Igualmente, la utilidad de los contratos inteligentes conjugada con la tarea notarial puede facilitar la formación de contratos que puedan requerir la intervención del notario.

24. Tur Faúndez (2018, pág. 81).

25. Vilalta Nicuesa (2019, pág. 119). Además, habrá que valorar el coste de la traducción del lenguaje formal al natural (Weber (2019, pág. 304), 26. Legerén-Molina (2018, pág. 227). Asimismo, será fácil probar la esencialidad del error cuando el consentimiento verse sobre la falta de comprensión del lenguaje informático.

27. Arnau Raventós (2015, pág. 433).

28. La empresa tecnológica de origen suizo Jur.lo ha desarrollado un smart contract que combina elementos del lenguaje natural y del formal. Véase el white paper de Jur.io: https://jur.io/wp-content/uploads/2019/05/jur-whitepaper-v.2.0.2.pdf [Fecha de consulta: 27 de abril de 2021].

29. Una muestra de ello la encontramos en: https://es.cointelegraph.com/news/the-blockchain-technology-could-replace-tasks-that-notaries-do [Fecha de consulta: 27 de abril de 2021].

30. Ibáñez Jiménez (2018, pág. 8).

31. Brancós i Nuñez (2017, pág. 51).

32. Rubio Gimeno (2020). 
Piénsese, por ejemplo, en el negocio jurídico testamentario $^{33}$. La compañía Mi Legado Digital ${ }^{34}$ ha desarrollado un modelo de testamento inteligente mediante la tecnología blockchain que conecta a todas las partes involucradas en el proceso sucesorio (herederos, notarios, aseguradoras, entidades financieras, registros, etc.). Por ello, consideramos idónea la tarea de propiciar una conjunción entre la fe pública notarial y la digitalización contractual.

\subsection{Los límites sustantivos del iContract}

Es bien sabido que una de las ventajas del lenguaje informático es su falta de ambigüedad ${ }^{35}$, aunque, por el contrario, es un lenguaje que poco servirá para programar un cambio de circunstancias, por ejemplo ${ }^{36}$. Además, los iContracts difícilmente podrán dar cabida a cláusulas que requieran ser interpretadas (por ejemplo, cuestiones relativas a la buena fe). Tampoco admiten, a causa de su carácter de ejecución automática, acuerdos de modificación del mismo ${ }^{37}$.

A pesar de ello, el desarrollo tecnológico exigirá dar respuesta a dichos inconvenientes: así, se ha propuesto establecer un derecho a «desistir» del contrato (a right to bail out), acordado ex ante por las partes, consistente en el pago de una cantidad de dinero ${ }^{38}$. Además, convendría también establecer la posibilidad de modificar determinados términos del contrato inteligente con el fin de proteger a la parte débil del mismo ${ }^{39}$. La renegociación del contrato se relaciona con el principio de buena fe contractual y el de conservación del contrato. Así, es la exigencia de un comportamiento recíproco basado en la lealtad, la hones- tidad y la consideración de los intereses de la otra parte la que actúa como reflejo del principio de buena $\mathrm{fe}^{40}$. Pues bien, técnicamente es posible prever en el propio código una posibilidad de modificación del iContract ${ }^{41}$.

En este sentido, el carácter inmutable propio de la cadena de bloques no debe determinar esta misma rigidez en el diseño y desarrollo de los contratos inteligentes ${ }^{42}$. Si no se permite dicha maleabilidad, el smart contract no debería calificarse como smart, sino más bien como dumb ${ }^{43}$.

\subsection{Smart arbitration: de la ejecución automática a la ejecución inteligente}

Se ha puesto ya de manifiesto el carácter de ejecución automática o determinista que rodea al contrato inteligente. Así, se ha señalado que los iContracts constituyen un instrumento que garantiza el cumplimiento del contrato y que, por tanto, reducen los costes asociados al incumplimiento del mismo ${ }^{44}$. De este modo, celebrado el contrato y comenzada la ejecución de las prestaciones, los sistemas programados impiden a las partes cualquier interferencia en el devenir del iter contractual.

El automatismo del iContract plantea el reto de prever algún mecanismo que permita abortar dicha ejecución ante la concurrencia de determinadas eventualidades ${ }^{45}$.

Sentado lo anterior, y con relación a la posibilidad de que haya una ejecución deficiente, podría plantearse un sistema de arbitraje que vehicule las reclamaciones; por ejemplo, pueden surgir cuestiones relativas a la capacidad

33. Gatteschi et al. (2019, pág. 47).

34. Así, https://www.milegadodigital.com/.

35. Durovic y Janssen (2019, pág. 76). Véase también Paech (2017, pág. 12)

36. Aunque ello se puede solucionar si se configura un oráculo que determine cuándo se produce un cambio sobrevenido e imprevisible de las circunstancias. Así, Tjong Tjin Tai (2019, pág. 96).

37. Raskin, M. (2017, págs. 326-327).

38. Meyer (2020, pág. 24).

39. Pasa y Dimatteo (2019, pág. 341).

40.Momberg Uribe (2015, págs. 74-75): «Derivado de la buena fe aparece aquí el deber de cooperación que se deben mutuamente los contratantes y que implica que ambos deben tener en consideración no solo su propio interés en el cumplimiento del contrato, sino también el de la contraparte, de manera de alcanzar el objetivo o fin común que se buscaba al celebrar el contrato, o lo que es lo mismo, los beneficios recíprocos derivados del acuerdo».

41. Así se expresa Weber (2019, págs. 308 y 309).

42. Raskin (2017, págs. 326-327).

43.Lipshaw (2018).

44.Ibáñez Jiménez (2018, pág. 110).

45. Así lo señala Paech (2017, págs. 12-13). 
de las partes para contratar, errores materiales en la introducción de los datos, etc. ${ }^{46} \mathrm{Al}$ respecto, huelga decir que la automatización progresiva de la ejecución en los contratos inteligentes conlleva que las situaciones en las que pueda predicarse un supuesto de incumplimiento contractual se relacionen, en su mayor medida, con fallos de la infraestructura digital empleada, y no (o no en su mayoría) con la voluntad de la otra parte de incumplir ${ }^{47},{ }^{48}$.

Tómese como ejemplo el propuesto por la tecnológica suiza Jur.lo, que ha desarrollado un mercado innovador que permite la creación de smart contracts. Asumiendo las limitaciones sustantivas que estos tienen, mediante lo que denomina «The Court Layer», ha desarrollado un mecanismo descentralizado de arbitraje que utiliza tecnología blockchain ${ }^{49}$.

Al hilo de lo que comentamos, nos resulta preferible el diseño de un mecanismo que permita detener dicha ejecución y que remita la decisión final a un tercero: un árbitro ${ }^{50}$. Fijémonos, por ejemplo, en el ámbito del transporte aéreo. La DA 2. a de la Ley 7/2017, de 2 de noviembre, por la que se incorpora al ordenamiento jurídico español la Directiva 2013/11/UE, del Parlamento Europeo y del Consejo, de 21 de mayo de 2013, relativa a la resolución alternativa de litigios en materia de consumo, señala que para la resolución de litigios de consumo sobre la aplicación de los Reglamentos de la UE en materia de protección de los usuarios del transporte aéreo será acreditada y notificada a la Comisión Europea una única entidad. Recientemente,

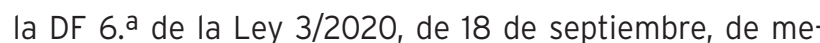
didas procesales y organizativas para hacer frente a la COVID-19 en el ámbito de la Administración de Justicia, ha modificado la DA 2. a de la Ley 7/2017 en el sentido de detallar, en parte, el procedimiento de resolución alternativa de los litigios en materia de transporte aéreo que deberá seguir la entidad acreditada para ello51. La decisión resultante, debidamente certificada por la entidad acreditada, tendrá la consideración de título ejecutivo extrajudicial. Como señala dicha DF, hasta que entre en vigor la orden de la persona titular del Ministerio de Transportes, Movilidad y Agenda Urbana, que regulará el procedimiento de resolución alternativa de los litigios, será la Agencia Estatal de Seguridad Aérea quien deberá acomodar su funcionamiento y procedimiento a lo previsto en esta ley con el fin de poder ser acreditada como entidad de resolución alternativa de litigios en materia de derechos de los usuarios del transporte aéreo ${ }^{52}, 53$.

Es preciso destacar, también, que entidades a las que hace referencia el art. 6.2 de la Ley 7/2017, como las Juntas Arbitrales de Consumo, pueden también dar cobertura a reclamaciones de consumo de todos los sectores económicos, siempre que ambas partes se hayan sometido voluntariamente a tales procedimientos.

El diseño del arbitraje que proponemos deberá superar el procedimiento proyectado en el art. 18 y en el art. 6.2 de la Ley $7 / 2017$, que exige que sea el consumidor quien plantee su pretensión ante el responsable del incumplimiento que origina la reclamación. Asimismo, según se prevé en la misma norma, será la compañía aérea o el gestor aeroportuario quien informará al consumidor de su adhesión o no a una entidad de resolución alternativa de litigios de consumo (ex art. 40 Ley $7 / 2017)^{54}$.

Se impone pues la necesidad de establecer (en el ejemplo que estamos proponiendo de iContracts relativos al transporte aéreo) un arbitraje de consumo obligatorio -posible si se establece con todas las garantías, tal y como apunta

46.Ibáñez Jiménez (2018, pág. 114).

47. Navas Navarro (2017, pág. 46)

48.Meyer (2020, pág. 23) plantea un sistema denominado «built-in dispute resolution».

49. Véase: https://jur.io/wp-content/uploads/2019/05/jur-whitepaper-v.2.0.2.pdf [Fecha de consulta: 27 de abril de 2021].

50.La modalidad propuesta sería de arbitraje electrónico (arts. 51 a 55 del RD 231/2008). En el marco europeo se ha proclamado la resolución de litigios en línea mediante el Reglamento (UE) 524/2013, del Parlamento Europeo y del Consejo, de 21 de mayo.

51. Dicha ley también modifica la Ley 21/2003, de 7 de julio, de seguridad aérea, añadiendo un nuevo artículo 62 bis y permitiendo la iniciación

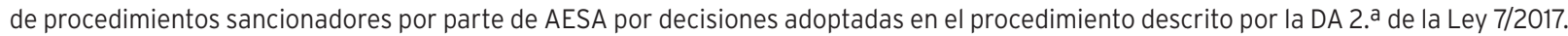

52. Véase el proyecto de orden del Ministerio de Fomento por el que se regula el procedimiento de resolución alternativa de litigios en materia de derechos de los usuarios de transporte: https://www.fomento.es/NR/rdonlyres/2D5A3CF7-46ED-433D-B127-4703B1410BDB/148613/OM_ADR_audienciaweb.pdf [Fecha de consulta: 27 de abril de 2021].

53. Viola Demestre (2019, págs. 51-81).

54.Gramunt Fombuena (2020a). 
Ia STC 1/2018, de 11 de enero de $2018^{55}$ La justificación de dicha obligatoriedad radica en la necesidad de proteger la parte débil de la relación: el consumidor (art. $51 \mathrm{CE}$ ). Asimismo, la posibilidad de sustituir el conocimiento de un asunto a los tribunales por la vía arbitral es perfectamente posible cuando se hace por disposición legal, y fundamentada en la obtención de finalidades claramente beneficiosas tales como la descarga del poder judicial, el acceso a un proceso más económico y, sobre todo, el reequilibrio de las posiciones contractuales ${ }^{56}$ En los supuestos en los que la compañía aérea considerase que la cancelación del vuelo, por ejemplo, tiene su origen en circunstancias extraordinarias que no hubieran podido evitarse incluso si se hubieran tomado todas las medidas razonables (Cdo. 12, Reglamento 534/2013), la ejecución del contrato quedaría en suspenso y se iniciaría el procedimiento arbitral ${ }^{57}$

\section{A modo de conclusión}

Según lo que hemos intentado poner de relieve, los contratos inteligentes, ante el desarrollo tecnológico actual en el campo de la contratación inteligente, quedan limitados a la definición legal de cuestiones poco ambiguas, claras y fácilmente trasladables a lenguaje formal. Ello es así porque los iContracts constituyen un instrumento que puede ser idóneo para anticipar condiciones y consecuencias del mismo negocio jurídico. Por el contrario, los contratos tradicionales juegan un papel preponderante en el terreno del ex post, permitiendo resolver aquellos conflictos que surgen del propio iter contractual. La imposibilidad de prevenir todas las consecuencias que pueden desencadenarse del devenir contractual refrenda a la contratación tradicional como óptima para el diseño de contratos de mayor complejidad. La contratación no puede en ningún caso prescindir de dicha complejidad que atañe a la negociación y, por lo tanto, ambos modelos convivirán, cada uno en su campo de juego.

Las observaciones anteriores confirman unas limitaciones que rodean a la contratación inteligente y que, por lo tanto, cuestionan, precisamente, dicha adjetivación relativa al intelecto. Deberá convenirse en que la contratación mediante lo que hemos Ilamado iContracts exigirá el respeto de los condicionantes impuestos por la normativa consumerista y por los límites resultantes de la protección de la parte débil, especialmente en lo relativo a la prestación del consentimiento.

Se impone también, finalmente, plantear una cuestión que merecerá una reflexión pausada en un futuro cual es la relativa al impacto que tendrá el desarrollo de la inteligencia artificial en la evolución del derecho contractual ${ }^{58}$

55 Además, la obligatoriedad del arbitraje podría limitarse a procedimientos de cuantía inferior a tres mil euros. En una línea parecida, véase el Reglamento núm. 861/2007 del Parlamento europeo y del Consejo, de 11 de julio de 2007, por el que se establece un proceso europeo de escasa cuantía que tiene como objetivo agilizar dichos litigios en asuntos transfronterizos.

56 La doctrina más autorizada ha defendido la necesidad de introducir un arbitraje de consumo obligatorio. Así, véase Gramunt Fombuena (2020b, págs. 1-14). La autora propone establecer un arbitraje de consumo potestativo para el consumidor, pero obligatorio para las empresas o profesionales en aquellos litigios la cuantía de los cuales no excediera los tres mil euros. La propuesta defiende que la adopción de la decisión arbitral deba ser sobre la base de la equidad, excepto que cualquiera de las dos partes solicite que se resuelva en derecho. Si el laudo debe resolverse en derecho, ambas partes podrían recurrir la decisión ante la jurisdicción civil; no, en cambio, en caso de que se resolviera en equidad.

57 Para ello sería necesario, de acuerdo con la legislación vigente, que en el diseño del IContract entre el consumidor y la compañía aérea se estableciera la cláusula relativa al arbitraje; es decir, la producción del supuesto previsto en el contrato (por ejemplo, cancelación del vuelo) automáticamente ejecutaría el reembolso del precio pagado a favor del cliente. Sin embargo, si la compañía aérea decidiera frenar la ejecución alegando circunstancias extraordinarias, el conflicto debería resolverse a través de la vía arbitral (recordemos que el art. 34.1 del RD 231/2008, de 15 de febrero, legitima únicamente al consumidor para iniciar el procedimiento arbitral). En el esquema propuesto, el consumidor ya habría aceptado la vía arbitral en el momento de la conclusión del contrato.

58 Micklitz et al. (2018, pág. 5.155) 


\section{Referencias bibliográficas}

ALLEN, J. G. (2018). «Wrapped and Stacked: Smart Contracts and the Interaction of Natural and Formal Language». ERCL, vol. 14, núm. 4 [en línea] DOI: https://doi.org/10.2139/ssrn.3297425 [Fecha de consulta: 27 de abril de 2021].

ARGELICH COMELLES, C. (2020). «Smart Contracts o Code is Law: soluciones legales para la robotización contractual». InDret, núm. 2 [en línea] [Fecha de consulta: 27 de abril de 2021].

ARNAU RAVENTÓS, L. (2015). «Formación de la voluntad: riesgos de captación, y “decisión con conocimiento de causa"». En: PEREÑA VICENTE, M.; DELGADO MARTíN, P. (2015). Nuevas orientaciones del Derecho civil en Europa. Pamplona: Thomson Reuters Aranzadi.

ARROYO AMAYUELAS, E. (2020). «La responsabilidad de los intermediarios en internet. ¿Puertos seguros a prueba de futuro?». Cuadernos de Derecho Transnacional, vol. 12, núm. 1.808 [en línea] DOI: https://doi.org/10.20318/cdt.2020.5225 [Fecha de consulta: 27 de abril de 2021].

BORGOGNO, O. (2019). «Usefulness and Dangers of Smart Contracts in Consumer Transactions». En: DIMATTEO, L. A.; CANNARSA, M.; PONCIBÒ, C. (2019). The Cambridge Handbook on Smart Contracts, Blockchain Technology and Digital Platforms. Cambridge: Cambridge University Press.

BRANCÓS I NUÑEZ, E. (2017). «Blockchain, función notarial y registro». El Notario del Siglo xxl: revista del Colegio Notarial de Madrid, núm. 71 [en línea] https://www.elnotario.es/hemeroteca/ revista-71/7325-blockchain-funcion-notarial-y-registro [Fecha de consulta: 27 de abril de 2021].

DE FILIPPI, P.; WRIGHT, A. (2018). Blockchain and the law, 3. ${ }^{a}$ ed. Cambridge, Massachusetts: Harvard University Press.

DÍAZ DÍAZ, E. (2019). «Una aplicación jurídica del Blockchain: los Smart Contracts». Actualidad Jurídica Aranzadi, núm. 951 [en línea] https://www.legaltoday.com/legaltech/novedades-legaltech/ una-aplicacion-juridica-del-blockchain-los-smart-contracts-2019-06-20/ [Fecha de consulta: 27 de abril de 2021].

DIMATTEO, L. A.; CANNARSA, M.; PONCIBÒ, C. (2019). The Cambridge Handbook on Smart Contracts, Blockchain Technology and Digital Platforms. Cambridge: Cambridge University Press.

DUROVIC, M.; JANSSEN, E. (2019). «Formation Smart Contracts under Contract Law». En: DIMATTEO, L. A., CANNARSA, M., PONCIBÒ, C. (2019). The Cambridge Handbook on Smart Contracts, Blockchain Technology and Digital Platforms, Cambridge: Cambridge University Press.

GATTESCHI et al. (2019). «Technology of smart contracts». En: DIMATTEO, L. A.; CANNARSA, M.; PONCIBÒ, C. (2019). The Cambridge Handbook on Smart Contracts, Blockchain Technology and Digital Platforms. Cambridge: Cambridge University Press.

GÓRRIZ LÓPEZ, C. (2017). «Tecnología blockchain y contratos inteligentes». En: NAVAS NAVARRO, S. et al. (2017). Inteligencia Artificial, Tecnología, Derecho, 1. a ed. Valencia: Tirant Lo Blanch.

GRAMUNT FOMBUENA, M. (2017). «La protección del contratante en contextos asimétricos». En: GRAMUNT FOMBUENA, M.; FLORENSA I TOMÀS, C. E. (2017). Codificación y reequilibrio de la asimetría negocial. Madrid: Dykinson.

GRAMUNT FOMBUENA, M. (2020a). «Obligatorietat versus voluntarietat en el sistema arbitral de consum» (ponencia). En: I Jornada Internacional sobre arbitratge. Barcelona: Agència Catalana de Consum, Generalitat de Catalunya.

GRAMUNT FOMBUENA, M. (2020b). «Perspectives sobre l'arbitratge obligatori». En: Jornada Virtual Internacional d'Arbitratge de Consum. Barcelona: Agència Catalana de Consum, Generalitat de Catalunya. 
IBÁÑEZ JIMÉNEZ, J. W. (2018). Derecho de Blockchain y de la tecnología de registros distribuidos, 1.a ed. Pamplona: Thomson Reuters Aranzadi.

IDELBERGER, F. (2018). «Smart Contracts as Contractual Networks». En: GRUNDMAN, S. (ed.) (2018). European Contract Law in the Digital Age. Cambridge: Intersentia.

LEGERÉN-MOLINA, A. (2019). «Retos jurídicos que plantea la tecnología de la cadena de bloques. Aspectos legales de blockchain». Revista de Derecho Civil, vol. VI, núm. 1 [en línea]: https://www.nreg. es/ojs/index.php/RDC/article/view/356

LEGERÉN-MOLINA, A. (2018). «Los contratos inteligentes en España. La disciplina de los smart contracts». Revista de Derecho civil, vol. V, núm. 2 [en línea] https://www.nreg.es/ojs/index.php/RDC/ article/view/320 [Fecha de consulta: 27 de abril de 2021].

LIPSHAW, J. M. (2018). «The Persistence of "Dumb" Contracts». Legal Studies Research Papers Series, Research Paper, vol. 18, núm. 11 [en línea] DOI: https://doi.org/10.2139/ssrn.3202484 [Fecha de consulta: 27 de abril de 2021].

LÓPEZ RODRÍGUEZ, A. M. (2021). «Ley aplicable a los smart contracts y lex crytographia». Cuadernos de Derecho Transnacional, vol. 13, núm. 1 [en línea] DOI: https://doi.org/10.20318/cdt.2021.5966 [Fecha de consulta: 27 de abril de 2021].

MEYER, O. (2020). «Stopping the Unstoppable: Termination and Unwinding of Smart Contracts». EUCML [en línea] https://papers.ssrn.com/sol3/papers.cfm?abstract_id=3537477 [Fecha de consulta: 27 de abril de 2021].

MICKLITZ, H.-W. et al. (2018). «Towards Consumer-Empowering Artificial Intelligence». Proceedings of the Twenty-Seventh International Joint Conference on Artificial Intelligence (IJCAI-2018) [en línea] DOI: http://dx.doi.org/10.24963/ijcai.2018/714 [Fecha de consulta: 27 de abril de 2021].

MIK, E. (2019). «Blockchains». En: DIMATTEO, L. A.; CANNARSA, M.; PONCIBÒ, C. (2019). The Cambridge Handbook on Smart Contracts, Blockchain Technology and Digital Platforms. Cambridge: Cambridge University Press.

MIK (2017). «Smart contracts: Terminology, technical limitations and real world complexity». Law, Innovation and Technology, vol. 9, núm. 2, pág. 285 [en línea] DOI: https://doi.org/10.2139/ssrn.3038406 [Fecha de consulta: 27 de abril de 2021].

MOMBERG URIBE, R. (2015). «Los efectos de la imprevisión contractual. Un análisis comparado». Revista de Derecho patrimonial, núm. 36.

NAKAMOTO, S. (2008). Bitcoin: a peer to peer electronic cash system [en línea] https://bitcoin.org/ bitcoin.pdf [Fecha de consulta: 27 de abril de 2021].

NAVAS NAVARRO, S. (2017). «Derecho e inteligencia artificial desde el diseño. Aproximaciones». En: NAVAS NAVARRO, S. et al. (2017). Inteligencia Artificial, Tecnología, Derecho, 1.a ed. Valencia: Tirant Lo Blanch.

PAECH, E. (2017). «The Governance of Blockchain Financial Networks». LSE Law, Society and Economic Working Papers, núm. 14. Londres: London School of Economics and Political Science [en línea] DOI: https://doi.org/10.2139/ssrn.2875487 [Fecha de consulta: 27 de abril de 2021].

PASA, B.; DIMATTEO, L. A. (2019). «Observations on the Impact of Technology on Contract Law». En: DIMATTEO, L. A.; CANNARSA, M.; PONCIBÒ, C. (2019). The Cambridge Handbook on Smart Contracts, Blockchain Technology and Digital Platforms. Cambridge: Cambridge University Press.

RASKIN, M. (2017). «The Law and Legality of Smart Contracts». Geo. L. Tech. Rev. 305 [en línea] https://georgetownlawtechreview.org/wp-content/uploads/2017/05/Raskin-1-GEO.-L.-TECH.-REV.-305-.pdf/ [Fecha de consulta: 27 de abril de 2021]. 
RUBIO GIMENO, G. (2020). «La contratación entre cónyuges o convivientes en los pactos sobre ruptura de pareja: ¿un negocio naturalmente asimétrico?». En: SERRANO DE NICOLÁS, Á. (dir.) (2020). Nuevas perspectivas iusprivatistas a los 40 años de la Constitución de 1978. Barcelona, Madrid, Buenos Aires, São Paulo: Marcial Pons.

SALVADOR CODERCH, P. (2020). «Contratos inteligentes y derecho del contrato». InDret, núm. 3 [en línea] https://indret.com/contratos-inteligentes-y-derecho-del-contrato/ [Fecha de consulta: 27 de abril de 2021].

SZABO, N. (1997). «Smart Contracts: Formalizing and Securing Relationships on Public Networks». First Monday, vol. 2, núm. 9 [en línea] DOI: https://doi.org/10.5210/fm.v2i9.548 [Fecha de consulta: 27 de abril de 2021].

SZABO, N. (1996). «Smart Contracts: Building Blocks for Digital Markets». Extropy, núm. 16 [en línea] https://www.fon.hum.uva.nl/rob/Courses/InformationInSpeech/CDROM/Literature/LOTwinterschool2006/szabo.best.vwh.net/smart_contracts_2.html [Fecha de consulta: 27 de abril de 2021].

TJONG TJIN TAI, E. (2019). «Challenges of Smart Contracts. Implementing Excuses». En: DIMATTEO, L. A.; CANNARSA, M.; PONCIBÒ, C. (2019). The Cambridge Handbook on Smart Contracts, Blockchain Technology and Digital Platforms. Cambridge: Cambridge University.

TUR FAÚNDEZ, C. (2018). Smart Contracts. Análisis jurídico, 1.a ed. Madrid: Reus Editorial.

VEGA MAZA, M. (2019). «El auge de blockchain y sus posibilidades reales de aplicación en los registros de las administraciones públicas». IDP. Revista de Internet, Derecho y Política, núm. 28 [en línea] DOI: https://doi.org/10.7238/idp.v0i28.3154 [Fecha de consulta: 27 de abril de 2021].

VILALTA NICUESA, A. E. (2019). Smart legal contracts y blockchain. La contratación inteligente a través de la tecnología blockchain, 1. ${ }^{a}$ ed. Madrid-Las Rozas: Wolters Kluwer.

VIOLA DEMESTRE, I. (2019). «EI procedimiento de acreditación de las entidades de resolución alternativa de litigios de consumo». En: VIOLA DEMESTRE, I.; PÉREZ DAUDÍ, V. (coord.); BARRAL VIÑALS, I. (dir.). El sistema de ADR/ODR en conflictos de consumo: aproximación crítica y prospección de futuro. Barcelona: Atelier.

WEBER, R. H. (2019). «Smart Contracts: Do we need New Legal Rules». En: DE FRANCESCHI, A.; SCHULZE, R. (2019). Digital Revolution-New Challenges for Law. Múnich: C. H. Beck-Nomos.

WERBACH, K. (2016). «Trust but verify: why the Blockchain needs the Law». Berkeley Technology Law Journal, vol. 33, núm. 2 [en línea]: https://lawcat.berkeley.edu/record/1128548

XALABARDER PLANTADA, R. (2006). «La responsabilidad de los prestadores de servicios en Internet (ISP) por infracciones de propiedad intelectual cometidas por sus usuarios». IDP. Revista de Internet, Derecho y Política, núm. 2.

ZHENG, Z. et al., (2020). «An overview on smart contracts: Challenges, advances and platforms». Future Generation Computer Systems, núm. 105 [en línea] DOI: https://doi.org/10.1016/j.future.2019.12.019 [Fecha de consulta: 27 de abril de 2021]. 


\title{
Cita recomendada
}

BARCELÓ COMPTE, Rosa (2021). «El impacto de la tecnología blockchain en la contratación privada: ¿hacia una contratación inteligente?». IDP. Revista de Internet, Derecho y Política, núm. 33 (octubre). UOC [Fecha de consulta: dd/mm/aa] http://dx.doi.org/10.7238/idp.v0i33.375171

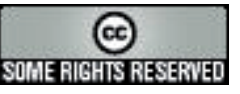

\begin{abstract}
Los textos publicados en esta revista están -si no se indica lo contrario- bajo una licencia Reconocimiento-Sin obras derivadas 3.0 España de Creative Commons. Puede copiarlos, distribuirlos y comunicarlos públicamente siempre que cite su autor y la revista y la institución que los publica (IDP. Revista de Internet, Derecho y Política; UOC); no haga con ellos obras derivadas. La licencia completa se puede consultar en: http://creativecommons.org/ licenses/by-nd/3.0/es/deed.es.
\end{abstract}

\section{Sobre la autora}

Rosa Barceló Compte

Investigadora posdoctoral de la Universidad de Barcelona

rbarcelo@ub.edu

Actualmente, la doctora Rosa Barceló es profesora de Derecho civil e investigadora posdoctoral de la Universidad de Barcelona.

Su trayectoria predoctoral se inició en 2014, cuando se integró en el grupo de investigación de Derecho y nuevas tecnologías de la Universidad de Barcelona, encabezado por las doctoras Mariló Gramunt y Gemma Rubio. En octubre de 2018 defendió la tesis titulada Ventaja injusta y protección de la parte débil del contrato, por la que recibió la calificación de excelente cum laude, y que a posteriori ha merecido el premio extraordinario de doctorado de la Facultad de Derecho. Finalmente, Marcial Pons publicó la monografía de la doctora Barceló, con el mismo título que su tesis doctoral, en 2019.

Su investigación se ha orientado en temas de reequilibrio económico del contrato, conservación del negocio jurídico y protección de la parte débil. También ha hecho investigación en el campo de la economía colaborativa, y actualmente sus estudios se centran en la contratación inteligente en el ámbito digital.

Ha llevado a cabo estancias de investigación en la Universidad de Utrecht y en la de Milán, y ha publicado varios artículos y colaboraciones científicas en obras colectivas.

Así mismo, ha impartido docencia en la UB y en la Universidad Rovira i Virgili de Tarragona. Actualmente, además de la UB, colabora como docente en la UOC.

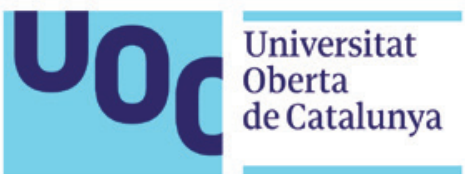

